\title{
Hybridization of Soft Expert Set with AHP in Decision Making
}

\author{
M. Saeed \\ Department of Mathematics \\ University of Management and \\ Technology \\ Lahore. Pakistan
}

\author{
Sahrish Mahmood \\ Department of Mathematics \\ University of Management and \\ Technology \\ Lahore. Pakistan
}

\author{
Hina Taufeeq \\ Department of Mathematics \\ University of Management and \\ Technology \\ Lahore. Pakistan
}

\begin{abstract}
In this note a typical problem of selecting manager is presented where soft expert set is indecisive. Because of this indecisiveness, hybridization of soft expert set with AHP technique has been developed, and solution is achieved. Furthermore, the effectiveness of result is verified by applying TOPSIS technique.
\end{abstract}

\section{Keywords}

Hybridization, Indecision, Soft Expert Set, Agree soft expert set, Disagree soft expert set, TOPSIS, RTD Matrix, CETD Matrix, AHP Technique

\section{INTRODUCTION}

Molodtsov[13] (1999) introduced soft set theory as a new mathematical tool to overcome on the uncertainties while modeling the issues in Medical Sciences, Computer sciences, Social Sciences, Engineering, Physics and Economics. In addition to this, Maji et al [11] also introduced an application of soft sets in decision making problems and comprehend the fundamental philosophy of soft set theory.

Over the past few years these methods are widely being used for several problems. Numerous researchers had done this study before and they created models too, to solve issues in decision making. Irony with the work is this that most of the models can tackle only one opinion or with one expert. And this creates hurdles for the user while obtaining data from the questionnaire.

Furthermore, Alkhazaleh and Salleh [17] stated soft expert set first then introduced a model for the users, by using this user can know the opinion of the experts without any operations.

Feng Kong And hongyan Liu [4] they aimed to find out the key factors that affect success in E-commerce using Fuzzy AHP, and give an evaluation method for E-commerce in order to help researchers and managers to determine the drawbacks and opportunities.

Jana Krejci et al [8] highlighted the necessity of applying the concept of constrained fuzzy arithematic instead of the concept of standard fuzzy arithmetic in a fuzzy extension of Analytic Hierarchy Process (AHP). Zulqarnain and Saeed [20] worked on interval valued fuzzy soft matrix (IVFSM) in decision making. They combined IVFSS with IVFS and soft sets, which is the consequence of Yang et al [19] Sabir Hussain[15] worked on soft expert topological spaces, Shabir, $\mathrm{M}$ and Naz, M[16] on soft topological spaces, Wand Y.J and Lee[18] worked on generalized TOPSIS for fuzzy multiple criteria group decision making. Gao [5-6], Ramik [14], Dodd [3], Hwang [7] and Linkov [10] works further on decision making problems.
Mainly the paper emphasizes is to attain a verdict for a everyday life problem by using soft expert set and the efficiency of the same issue is demonstrated with the help of Combined Effect Time Dependent Data (CETD) Matrix and it is checked for stability via Analytic Hierarchy Process (AHP). Finally, the results are verified by TOPSIS technique.

\section{PRELIMINARIES}

\subsection{Definition: (Soft Expert Set)}

A pair $(F, A)$ is called a soft expert set over $U$, where $F$ is a mapping given by

$$
F: A \rightarrow P(U)
$$

Where $\mathrm{P}(\mathrm{U})$ denotes the power set of $\mathrm{U}$.

\subsection{Definition: (Agree-Soft expert Set)}

An agree soft expert set $(F, A)_{1}$ over $\mathrm{U}$ is a soft expert subset of $(\mathrm{F}, \mathrm{A})$ is defined as

$$
(F, A)_{1}=\left\{F_{1} e: e \in E * X * 1\right\}
$$

\subsection{Definition: (Disagree-Soft Expert}

Set)

An disagree soft expert set $(F, A)_{0}$ over $\mathrm{U}$ is a soft expert subset of $(\mathrm{F}, \mathrm{A})$ is defined as

$$
(F, A)_{0}=\left\{F_{0}(e): e \in E * X * 0\right\}
$$

\subsection{Definition: (AHP)}

The Analytic Hierarchy Process (AHP) is a multi-criteria decision making method allows decision makers to model a complex problem in a hierarchical structure, showing the relationships of the goal, objectives (criteria), and alternatives.

\section{DESCRIPTION OF PROPOSED METHODOLOGY}

The proposed methodology requires making a decision for selection of the Manager. This was initially done by applying Soft Expert Set on the opinions of experts. Steps of Soft

Expert set are as follows:

- Assigning weights to parameters

- Construction of Agree Matrix

- Construction of Average Time Dependent (ATD) Matrix

- Construction of Refined Time Dependent(RTD) Matrix

- Construction of CETD Matrix

- Sum of column Matrix

- Observation of outcome and arrive at result.

The implementation of Soft Expert Set resulted in multiple decisions instead of one. Arriving at a situation where two persons out of four were ineligible. So to select between two 
remaining persons, the AHP technique is used. Steps of AHP are as follows [12]:

- Make a pair wise comparison matrix for criteria. In this study, a three point scale has been used as previous studies. because it has been demonstrated that most responders do not use more than three judgments (equal, more, much more) and lay users reported puzzled at a moment of using a more complex scale.

- Determine the relative normalized weight $\left(W_{j}\right)$ of each criteria/sub-criterion by normalizing the geometric mean of the rows in the comparison matrix:

$$
\begin{aligned}
& G M_{j}=\prod_{j=1}^{N} a_{i j}{ }^{1 / N} \\
& W_{j}=G M_{j} / \sum_{j=1}^{N} G M_{j}
\end{aligned}
$$

\section{AN IMPLEMENTATION OF SOFT \\ EXPERT SET}

In this section, the implementation of soft expert set in the problem of decision making is presented. The problem that is to be considered is as follows:

Assume that a company wants to hire Manager. There are four nominees, who, from the universe $U=\left\{s_{1}, s_{2}, s_{3}, s_{4}\right\}$. Let $X=\{p, q, r\}$ be a set of expert members. These experts consider a set of parameters $E=\left\{e_{1}, e_{2}, e_{3}\right\}$ where the parameter $i,(i=1,2,3)$ stands for the Language Test, Professional Test and Academic Record respectively.

\subsection{Decision Making Team}

The expert team was formed by:

- $\quad \mathrm{p}=$ Administrative head of the entire company (with 15 years of experience)

- $\mathrm{q}=$ Head of HRM department (with 10 years of experience)

- $\quad r=$ Managing Director (MD) under whose supervision the managers have to work to achieve certain goals. (with 5 years of experience)

\subsection{Description of Criteria}

- $\quad e_{1}=$ Language Test is described as the command of a person over language. The person is supposed to be exceptionally fluent in required linguistics.

- $\quad e_{2}=$ Professional test is described as the assessment of professional skills that a person has acquired. How well he/she adjusts with the enviourment as well as copes up with the situation of stress.

- $\quad e_{3}=$ Acedemic Record is the compiled form of the person's educational achievements i.e. acquired degrees, distinctions/grades (percentage, cpga etc)

Assume:

$$
\begin{gathered}
(F, Z)=\left\{\left(\left(e_{1}, p, 1\right),\left\{s_{1}, s_{2}\right\}\right),\left(\left(e_{1}, q, 1\right),\left\{s_{3}, s_{4}\right\}\right),\right. \\
\left(\left(e_{1}, r, 1\right),\left\{s_{1}, s_{2}, s_{3}\right\}\right), \\
\left(\left(e_{2}, p, 1\right),\left\{s_{3}, s_{4}\right\}\right),\left(\left(e_{2}, q, 1\right),\left\{s_{1}, s_{2}\right\}\right), \\
\left(\left(e_{2}, r, 1\right),\left\{s_{4}\right\}\right), \\
\left(\left(e_{3}, p, 1\right),\left\{s_{1}, s_{3}\right\}\right),\left(\left(e_{3}, q, 1\right),\left\{s_{1}, s_{2}, s_{4}\right\}\right), \\
\left(\left(e_{3}, r, 1\right),\left\{s_{1}, s_{4}\right\}\right), \\
\left(\left(e_{1}, p, 0\right),\left\{s_{3}, s_{4}\right\}\right),\left(\left(e_{2}, q, 0\right),\left\{s_{1}, s_{2}\right\}\right),
\end{gathered}
$$

$$
\begin{gathered}
\left(\left(e_{1}, r, 0\right),\left\{s_{4}\right\}\right), \\
\left(\left(e_{2}, p, 0\right),\left\{s_{1}, s_{2}\right\}\right),\left(\left(e_{2}, q, 0\right),\left\{s_{3}, s_{4}\right\}\right), \\
\left(\left(e_{2}, r, 0\right),\left\{s_{1}, s_{2}, s_{3}\right\}\right), \\
\left(\left(e_{3}, p, 0\right),\left\{s_{2}, s_{4}\right\}\right),\left(\left(e_{3}, q, 0\right),\left\{s_{3}\right\}\right), \\
\left.\left(\left(e_{3}, r, 0\right),\left\{s_{2}, s_{3}\right\}\right)\right\}
\end{gathered}
$$

This states that the decision makers $\mathrm{p}, \mathrm{q} \& \mathrm{r} \operatorname{choose}\left\{s_{1}, s_{2}\right\}$, $\left\{s_{3}, s_{4}\right\} \&\left\{s_{1}, s_{2}, s_{3}\right\}$ respectively by judging $\left\{s_{1}, s_{2}, s_{3}, s_{4}\right\}$ on the criteria $e_{1}$ i.e. Language test.

It can also be said that decision makers $\mathrm{p}, \mathrm{q} \& \mathrm{r}$ did not choose $\left\{s_{3}, s_{4}\right\},\left\{s_{1}, s_{2}\right\} \&\left\{s_{4}\right\}$ respectively on the criteria $e_{1}$ i.e. Language test.

1 shows the eligibility criteria of decision maker where as 0 shows ineligibility criteria.

Now, in order to make a decision, i.e. to choose one person out of others for Manager Post, the parameters are given some weight, say

$$
e_{1}=0.5, e_{2}=0.7, e_{3}=1
$$

The result can be made by the use of agree matrix as follows:

$$
\left(\begin{array}{llll}
2 & 2 & 2 & 1 \\
1 & 1 & 1 & 2 \\
3 & 1 & 1 & 2
\end{array}\right)
$$

This matrix is made with the problem described in $(\mathrm{F}, \mathrm{Z})$. The Average Time Dependent Data (ATD) Matrix obtained is

$$
\left(\begin{array}{cccc}
4 & 4 & 4 & 2 \\
1.43 & 1.43 & 1.43 & 2.86 \\
3 & 1 & 1 & 2
\end{array}\right)
$$

To obtain the Refined Time Dependent Data (RTD) Matrix [2] we use.

$$
\begin{aligned}
& \text { if } a_{i j} \leq\left(\mu_{j}-\alpha * \sigma_{j}\right) \text { Then } e_{i j}=-1 \\
& \text { if } a_{i j} \in\left(\mu_{j}-\alpha * \sigma_{j}, \mu_{j}+\alpha * \sigma_{j}\right) \text { Then } e_{i j}=0 \\
& \text { if } a_{i j} \geq\left(\mu_{j}-\alpha * \sigma_{j}\right) \text { Then } e_{i j}=+1
\end{aligned}
$$

With values of $\alpha$ as follows $0.25,0.50,1$ the following RTD's were obtained.

For $\alpha=0.25$

$$
\left(\begin{array}{cccc}
1 & 1 & 1 & -1 \\
-1 & -1 & -1 & 1 \\
0 & -1 & -1 & -1
\end{array}\right)
$$

For $\alpha=0.50$

$$
\left(\begin{array}{cccc}
1 & 1 & 1 & -1 \\
-1 & -1 & -1 & 1 \\
0 & -1 & -1 & 0
\end{array}\right)
$$

For $\alpha=0.75$

$$
\left(\begin{array}{cccc}
1 & 1 & 1 & 0 \\
-1 & 0 & 0 & 1 \\
0 & -1 & -1 & 0
\end{array}\right)
$$

The CETD matrix formed with the above obtained RTD is

$$
\left(\begin{array}{cccc}
3 & 3 & 3 & -2 \\
-3 & -2 & -2 & 3 \\
0 & -3 & -3 & -1
\end{array}\right)
$$


The sum of above column matrix is $\left(\begin{array}{llll}0 & -2 & -2 & 0\end{array}\right)$. This matrix gives the outcome that persons at $s_{2}$ and $s_{3}$ positions cannot be opted for the manager post.

If, considered the same problem with different weights of parameter say

$$
e_{1}=0.6, e_{2}=0.4, e_{3}=1
$$

Then, the agree matrix will be same but the Average Time Dependant Data (RTD) Matrix obtained is

$$
\left(\begin{array}{cccc}
3.33 & 3.33 & 3.33 & 1.67 \\
2.5 & 2.5 & 2.5 & 5 \\
3 & 1 & 1 & 2
\end{array}\right)
$$

The column matrix is $\left(\begin{array}{llll}0 & 0 & 0 & -2\end{array}\right)$. (This is done just for the purpose of comparison between answer of two weights selected) This matrix gives the result that person at $s_{4}$ position cannot be opted for the manager post. Now, check for selection of the persons at $s_{1}, s_{2}$ and $s_{3}$ positions by applying AHP.

Here it can be briefly said that soft expert set did not choose any person, rather it eliminated one person in this particular case. To make a choice further AHP technique is applied.

\section{HYBRIDIZATION WITH AHP}

\section{TECHNIQUE}

AHP is scalable. AHP is usually used to reduce the complexities of decision making in a certain and definite way. This method support (or else is applicable) for both single person also to make a group decision. This method does not necessarily need the presence of any specialist. [1]

The AHP is a powerful and flexible Multi Criteria Decision Making tool for dealing with complex problems where both qualitative and quantitative aspects are taken into consideration. [9]

The problem of selecting a best Manager is applied again with this technique to verify the results obtained from soft expert set. The persons are compared using the pair wise comparison scale for AHP preference. The following tables are framed with Saaty's scale for each of the parameter.

Table 1. Comparison Table For $e_{1}$

\begin{tabular}{|c|l|l|l|l|}
\hline Parameter $\boldsymbol{e}_{\mathbf{1}}$ & $\boldsymbol{s}_{\mathbf{1}}$ & $\boldsymbol{s}_{\mathbf{2}}$ & $\boldsymbol{s}_{\mathbf{3}}$ & $\boldsymbol{s}_{\mathbf{4}}$ \\
\hline $\boldsymbol{s}_{\mathbf{1}}$ & 1 & 1 & 1 & 2 \\
\hline $\boldsymbol{s}_{\mathbf{2}}$ & 1 & 1 & 1 & 2 \\
\hline $\boldsymbol{s}_{\mathbf{3}}$ & 1 & 1 & 1 & 2 \\
\hline $\boldsymbol{s}_{\mathbf{4}}$ & $1 / 2$ & $1 / 2$ & $1 / 2$ & 1 \\
& & & & \\
\hline
\end{tabular}

Table 2. Comparison Table For $\boldsymbol{e}_{2}$

\begin{tabular}{|c|c|c|c|c|}
\hline Parameter $\boldsymbol{e}_{\mathbf{2}}$ & $\boldsymbol{s}_{\mathbf{1}}$ & $\boldsymbol{s}_{\mathbf{2}}$ & $\boldsymbol{s}_{\mathbf{3}}$ & $\boldsymbol{s}_{\mathbf{4}}$ \\
\hline $\boldsymbol{s}_{\mathbf{1}}$ & 1 & 1 & 1 & $1 / 2$ \\
\hline $\boldsymbol{s}_{\mathbf{2}}$ & 1 & 1 & 1 & $1 / 2$ \\
\hline $\boldsymbol{s}_{\mathbf{3}}$ & 1 & 1 & 1 & $1 / 2$ \\
\hline $\boldsymbol{s}_{\mathbf{4}}$ & 2 & 2 & 2 & 1 \\
\hline
\end{tabular}

Table 3. Comparison Table For $\boldsymbol{e}_{3}$

\begin{tabular}{|c|c|c|c|c|}
\hline Parameter $\boldsymbol{e}_{\mathbf{3}}$ & $\boldsymbol{s}_{\mathbf{1}}$ & $\boldsymbol{s}_{\mathbf{2}}$ & $\boldsymbol{s}_{\mathbf{3}}$ & $\boldsymbol{s}_{\mathbf{4}}$ \\
\hline $\boldsymbol{s}_{\mathbf{1}}$ & 1 & 3 & 3 & 2 \\
\hline $\boldsymbol{s}_{\mathbf{2}}$ & $1 / 3$ & 1 & 1 & $1 / 2$ \\
\hline $\boldsymbol{s}_{\mathbf{3}}$ & $1 / 3$ & 1 & 1 & $1 / 2$ \\
\hline $\boldsymbol{s}_{\mathbf{4}}$ & $1 / 2$ & 2 & 2 & 1 \\
\hline
\end{tabular}

The above tables are used to find the Eigen vector $A_{i j}$ by dividing the value using the column sum.

Table 4. Average table for parameter $e_{1}$

\begin{tabular}{|c|c|c|c|c|c|}
\hline $\begin{array}{c}\text { Parameter } \\
\boldsymbol{e}_{\mathbf{1}}\end{array}$ & $\boldsymbol{s}_{\mathbf{1}}$ & $\boldsymbol{s}_{\mathbf{2}}$ & $\boldsymbol{s}_{\mathbf{3}}$ & $\boldsymbol{s}_{\mathbf{4}}$ & $\begin{array}{c}\text { Avera } \\
\text { ge } \\
A_{i j}\end{array}$ \\
\hline $\boldsymbol{s}_{\mathbf{1}}$ & $\begin{array}{c}0.285 \\
7\end{array}$ & $\begin{array}{c}0.285 \\
7\end{array}$ & $\begin{array}{c}0.28 \\
57\end{array}$ & 0.2857 & $\begin{array}{c}0.285 \\
7\end{array}$ \\
\hline $\boldsymbol{s}_{\mathbf{2}}$ & $\begin{array}{c}0.285 \\
7\end{array}$ & $\begin{array}{c}0.285 \\
7\end{array}$ & $\begin{array}{c}0.28 \\
57\end{array}$ & 0.2857 & $\begin{array}{c}0.285 \\
7\end{array}$ \\
\hline $\boldsymbol{s}_{\mathbf{3}}$ & $\begin{array}{c}0.285 \\
7\end{array}$ & $\begin{array}{c}0.285 \\
7\end{array}$ & $\begin{array}{c}0.28 \\
57\end{array}$ & 0.2857 & $\begin{array}{c}0.285 \\
7\end{array}$ \\
\hline $\boldsymbol{s}_{\mathbf{4}}$ & 0.142 & 0.142 & 0.14 & 0.1428 & 0.142 \\
& 8 & 8 & 28 & & 8 \\
\hline
\end{tabular}

Above table is the average table for parameter $e_{1}$, which is obtained from comparison table of $e_{1}$. This table will be used to find Eigen values for $e_{1}$.

Table 5. Average table for parameter $e_{2}$

\begin{tabular}{|c|c|c|c|c|c|}
\hline $\begin{array}{c}\text { Parameter } \\
\boldsymbol{e}_{\mathbf{2}}\end{array}$ & $\boldsymbol{s}_{\mathbf{1}}$ & $\boldsymbol{s}_{\mathbf{2}}$ & $\boldsymbol{s}_{\mathbf{3}}$ & $\boldsymbol{s}_{\mathbf{4}}$ & $A_{i j}$ \\
\hline $\boldsymbol{s}_{\mathbf{1}}$ & 0.2 & 0.2 & 0.2 & 0.2 & 0.2 \\
\hline $\boldsymbol{s}_{\mathbf{2}}$ & 0.2 & 0.2 & 0.2 & 0.2 & 0.2 \\
\hline $\boldsymbol{s}_{\mathbf{3}}$ & 0.2 & 0.2 & 0.2 & 0.2 & 0.2 \\
\hline $\boldsymbol{s}_{\mathbf{4}}$ & 0.4 & 0.4 & 0.4 & 0.4 & 0.4 \\
\hline
\end{tabular}


Above table is the average table for parameter $e_{2}$, which is obtained from comparison table of $e_{2}$. This table will be used to find Eigen values for $\boldsymbol{e}_{2}$.

Table 6. Average table for parameter $e_{3}$

\begin{tabular}{|c|c|c|c|c|l|}
\hline $\begin{array}{l}\text { Parameter } \\
\boldsymbol{e}_{\mathbf{3}}\end{array}$ & $\boldsymbol{s}_{\mathbf{1}}$ & $\boldsymbol{s}_{\mathbf{2}}$ & $\boldsymbol{s}_{\mathbf{3}}$ & $\boldsymbol{s}_{\mathbf{4}}$ & $\begin{array}{l}\text { Avera } \\
\text { ge } \\
\boldsymbol{A}_{i j}\end{array}$ \\
\hline $\boldsymbol{s}_{\mathbf{1}}$ & 0.4615 & 0.4285 & 0.4285 & 0.5 & $\begin{array}{l}0.454 \\
6\end{array}$ \\
\hline $\boldsymbol{s}_{\mathbf{2}}$ & 0.1538 & 0.1428 & 0.1428 & 0.125 & 0.141 \\
& & & & & 1 \\
\hline $\boldsymbol{s}_{\mathbf{3}}$ & 0.1538 & 0.1428 & 0.1428 & 0.125 & 0.141 \\
& & & & & 1 \\
\hline $\boldsymbol{s}_{\mathbf{4}}$ & 0.23076 & 0.2857 & 0.2857 & 0.25 & 0.263 \\
& & & & & 04 \\
\hline
\end{tabular}

Above table is the average table for parametere $e_{3}$, which is obtained from comparison table of $e_{3}$. This table will be used to find Eigen values for $\boldsymbol{e}_{3}$.

The corresponding Eigen values are:

\begin{tabular}{ll}
- & For $e_{1}(1.1428,1.1428,1.1428,0.5714)$ \\
- & For $e_{2}(0.8,0.8,0.8,1.6)$ \\
\hline & For $e_{3}(1.827,0.561,0.565,1.195)$
\end{tabular}

The Eigen values suggest that $s_{l}$ is best choice for the manager post.

\section{VERIFICATION WITH TOPSIS}

Parameter table of AHP method is used:

Table 7. For $e_{1}$

\begin{tabular}{|c|c|c|c|c|c|}
\hline $\begin{array}{c}\text { Parameter } \\
\boldsymbol{e}_{\mathbf{3}}\end{array}$ & $\boldsymbol{s}_{\mathbf{1}}$ & $\boldsymbol{s}_{\mathbf{2}}$ & $\boldsymbol{s}_{\mathbf{3}}$ & $\boldsymbol{s}_{\mathbf{4}}$ & $A_{i j}$ \\
\hline $\boldsymbol{s}_{\mathbf{1}}$ & 0.2857 & 0.2857 & 0.2857 & 0.2857 & 0.2857 \\
\hline $\boldsymbol{s}_{\mathbf{2}}$ & 0.2857 & 0.2857 & 0.2857 & 0.2857 & 0.2857 \\
\hline $\boldsymbol{s}_{\mathbf{3}}$ & 0.2857 & 0.2857 & 0.2857 & 0.2857 & 0.2857 \\
\hline $\boldsymbol{s}_{\mathbf{4}}$ & 0.1428 & 0.1428 & 0.1428 & 0.1428 & 0.1428 \\
\hline
\end{tabular}

Here another technique is applied, known as TOPSIS. This method is applied on AHP just for verification of the results. In TOPSIS average is used as weights. The TOPSIS method includes following steps:

\subsection{Step 1}

Table 8. Calculate $\left(\sum x_{i j}{ }_{i j}\right)^{\frac{1}{2}}$ for each column

\begin{tabular}{|c|c|c|c|c|}
\hline $\boldsymbol{s}_{\mathbf{i}}^{\mathbf{2}}$ & $\boldsymbol{s}_{\mathbf{1}}$ & $\boldsymbol{s}_{\mathbf{2}}$ & $\boldsymbol{s}_{\mathbf{3}}$ & $\boldsymbol{s}_{\mathbf{4}}$ \\
\hline $\boldsymbol{s}_{\mathbf{1}}$ & 0.08162 & 0.08162 & 0.08162 & 0.08162 \\
\hline $\boldsymbol{s}_{\mathbf{2}}$ & 0.08162 & 0.08162 & 0.08162 & 0.08162 \\
\hline $\boldsymbol{s}_{\mathbf{3}}$ & 0.08162 & 0.08162 & 0.08162 & 0.08162 \\
\hline $\boldsymbol{s}_{\mathbf{4}}$ & 0.02039 & 0.02039 & 0.02039 & 0.02039 \\
\hline$\left(\sum x^{2}{ }_{i j}\right)$ & 0.26525 & 0.26525 & 0.26525 & 0.26525 \\
\hline$\left(\sum x^{2}{ }_{i j}\right)^{\frac{1}{2}}$ & 0.51502 & 0.51502 & 0.51502 & 0.51502 \\
\hline
\end{tabular}

\subsection{Step 2}

Table 9. Divide each column by $\left(\sum x^{2}{ }_{i j}\right)^{\frac{1}{2}}$ to get $r_{i j}$

\begin{tabular}{|l|l|l|l|l|}
\hline & \multicolumn{1}{|c|}{$\boldsymbol{s}_{\mathbf{1}}$} & \multicolumn{1}{|c|}{$\boldsymbol{s}_{\mathbf{2}}$} & \multicolumn{1}{c|}{$\boldsymbol{s}_{\mathbf{3}}$} & \multicolumn{1}{c|}{$\boldsymbol{s}_{\mathbf{4}}$} \\
\hline $\boldsymbol{s}_{\mathbf{1}}$ & 0.5547 & 0.5547 & 0.5547 & 0.5547 \\
\hline $\boldsymbol{s}_{\mathbf{2}}$ & 0.5547 & 0.5547 & 0.5547 & 0.5547 \\
\hline $\boldsymbol{s}_{\mathbf{3}}$ & 0.5547 & 0.5547 & 0.5547 & 0.5547 \\
\hline $\boldsymbol{s}_{\mathbf{4}}$ & 0.2773 & 0.2773 & 0.2773 & 0.2773 \\
\hline
\end{tabular}

\subsection{Step 3}

Table 10. Multiply each column by $w_{i j}$ to get $v_{i j}$

\begin{tabular}{|l|l|l|l|l|}
\hline & \multicolumn{1}{|c|}{$\boldsymbol{s}_{\mathbf{1}}$} & \multicolumn{1}{|c|}{$\boldsymbol{s}_{\mathbf{2}}$} & $\boldsymbol{s}_{\mathbf{3}}$ & $\boldsymbol{s}_{\mathbf{4}}$ \\
\hline $\boldsymbol{s}_{\mathbf{1}}$ & 0.15848 & 0.15848 & 0.15848 & 0.0792 \\
\hline $\boldsymbol{s}_{\mathbf{2}}$ & 0.15848 & 0.15848 & 0.15848 & 0.0792 \\
\hline $\boldsymbol{s}_{\mathbf{3}}$ & 0.15848 & 0.15848 & 0.15848 & 0.0792 \\
\hline $\boldsymbol{s}_{\mathbf{4}}$ & 0.07922 & 0.07922 & 0.07922 & 0.0396 \\
\hline
\end{tabular}

\subsection{Step 4}

Determine Ideal Solution $A^{*}$ from the step 3

$$
A^{*}=\{0.15848,0.15848,0.15848,0.0792\}
$$

\subsection{Step 5}

Determine Ideal Solution $A^{\prime}$ from the step 3

$$
A^{\prime}=\{0.07922,0.07922,0.07922,0.0396\}
$$

\subsection{Step 6}

Calculate separation from the ideal Solution

$A^{*}=\{0.15848,0.15848,0.15848,0.0792\}$

$$
S^{*}=\left[\sum\left(v j j^{*}-v i j\right)^{2}\right]^{\frac{1}{2}}
$$

For each row 
Table 11. Separation from ideal solution

\begin{tabular}{|l|l|l|l|l|}
\hline & \multicolumn{1}{|c|}{$\boldsymbol{s}_{\mathbf{1}}$} & \multicolumn{1}{c|}{$\boldsymbol{s}_{\mathbf{2}}$} & \multicolumn{1}{c|}{$\boldsymbol{s}_{\mathbf{3}}$} & \multicolumn{1}{c|}{$\boldsymbol{s}_{\mathbf{4}}$} \\
\hline $\boldsymbol{s}_{\mathbf{1}}$ & 0 & 0 & 0 & 0 \\
\hline $\boldsymbol{s}_{\mathbf{2}}$ & 0 & 0 & 0 & 0 \\
\hline $\boldsymbol{s}_{\mathbf{3}}$ & 0 & 0 & 0 & 0 \\
\hline $\boldsymbol{s}_{\mathbf{4}}$ & $\begin{array}{l}(0.07922 \\
-0.15848)^{2}\end{array}$ & $\begin{array}{l}(0.07922 \\
-0.15848)^{2}\end{array}$ & $\begin{array}{l}(0.07922 \\
-0.15848)^{2}\end{array}$ & $(-0.0396)^{2}$ \\
\hline
\end{tabular}

\section{$6.7 \quad$ Step 7}

Table 12. Calculate separation from the ideal $S_{i}^{*}$

\begin{tabular}{|c|c|c|}
\hline & $\sum\left(v^{*}{ }_{j j}-v_{i j}\right)^{2}$ & $S_{i}^{*}=\left\lceil\left.\sum\left(v^{*}{ }_{j j}-v_{i j}\right)^{2}\right|^{\frac{1}{2}}\right.$ \\
\hline$s_{1}$ & 0 & 0 \\
\hline$s_{2}$ & 0 & 0 \\
\hline$s_{3}$ & 0 & 0 \\
\hline$s_{4}$ & 0.020415 & 0.142881 \\
\hline
\end{tabular}

\subsection{Step 8}

Calculate separation from the negative ideal solution

$$
\begin{gathered}
A^{\prime}=\{0.07922,0.07922,0.07922,0.0396\} \\
S_{i}^{\prime}=\left\lceil\sum\left(\boldsymbol{v}^{*}{ }_{j}-\boldsymbol{v}_{\boldsymbol{i} \boldsymbol{j}}\right)^{2}\right\rceil^{\frac{1}{2}}
\end{gathered}
$$

Table 13. For each row

\begin{tabular}{|l|l|l|l|l|}
\hline & \multicolumn{1}{|c|}{$\boldsymbol{s}_{\mathbf{1}}$} & \multicolumn{1}{c|}{$\boldsymbol{s}_{\mathbf{2}}$} & $\boldsymbol{s}_{\mathbf{3}}$ & $\boldsymbol{s}_{\mathbf{4}}$ \\
\hline $\boldsymbol{s}_{\mathbf{1}}$ & 0.0062821 & 0.0062821 & 0.0062821 & 0.00156816 \\
\hline $\boldsymbol{s}_{\mathbf{2}}$ & 0.0062821 & 0.0062821 & 0.0062821 & 0.00156816 \\
\hline $\boldsymbol{s}_{\mathbf{3}}$ & 0.0062821 & 0.0062821 & 0.0062821 & 0.00156816 \\
\hline $\boldsymbol{s}_{\mathbf{4}}$ & 0 & 0 & 0 & 0 \\
\hline
\end{tabular}

\subsection{Step 9}

Table 14. Calculate separation from the ideal $S_{i}^{\prime}$

\begin{tabular}{|c|c|c|}
\hline & $\sum\left(v^{*}{ }_{j j}-v_{i j}\right)^{2}$ & $S_{i}^{*}=\left\lceil\sum\left(v^{*}{ }_{j j}-v_{i j}\right)^{2}\right]^{\frac{1}{2}}$ \\
\hline$s_{1}$ & 0.02041 & 0.142879 \\
\hline$s_{2}$ & 0.02041 & 0.142879 \\
\hline$s_{3}$ & 0.02041 & 0.142879 \\
\hline$s_{4}$ & 0 & 0 \\
\hline
\end{tabular}

\subsection{Step 10}

Calculate the relative closeness to the ideal solution $C_{i}^{*}$

$$
C_{i}^{*}=\frac{S_{i}^{\prime}}{S_{i^{*}}+S_{i}^{\prime}}
$$

Table 15. Relative closeness to ideal solution

\begin{tabular}{|c|c|c|}
\hline & $\boldsymbol{C}_{i}^{*}=\frac{\boldsymbol{S}_{\boldsymbol{i}}^{\prime}}{\boldsymbol{S}_{\boldsymbol{i}^{*}}+\boldsymbol{S}_{\boldsymbol{i}}^{\prime}}$ & $\boldsymbol{C}_{\boldsymbol{i}}^{*}$ \\
\hline $\boldsymbol{s}_{\mathbf{1}}$ & $\frac{0.142879}{0.142879}$ & 1 \\
\hline $\boldsymbol{s}_{\mathbf{2}}$ & 1 & 1 \\
\hline $\boldsymbol{s}_{3}$ & 1 & 1 \\
\hline $\boldsymbol{s}_{\mathbf{4}}$ & 1 & 0 \\
\hline
\end{tabular}

The results show that $s_{1}, s_{2}, s_{3}$ can be opted for the post.

- $\quad$ Similarly, we applied the steps on parameter $e_{2}$

- And the results show that $s_{4}$ was most suitable person.

- $\quad \&$ for parametere $e_{3}, s_{1}$ was suitable for the post.

- Since $s_{1}$ has satisfied the criteria in two parameters

- Hence $s_{1}$ will be selected in TOPSIS method.

\section{CONCLUSION}

In this paper, decision making problem was analyzed using soft expert set, through which results were indecisive. Consequently decision could not be made, and then AHP technique was applied to avoid uncertainty. Finally, the results were verified by TOPSIS method. When soft expert set is hybridized with AHP it reduces cost. TOPSIS technique verifies that it does not affect the final outcome. In future hybridization of soft expert set with other methods can be done, and their results can be further analyzed.

\section{REFERENCES}

[1] Aktas, H. and Cagman, N., 2007 soft Sets and Soft Groups Inf.Sci. 2726-2735.

[2] Arockiarni, I. and Arokia, A. Lancy., 2013 Multicriteria Decision making problem with soft expert set, International Journal of Computer applications, vol 78

[3] Dodd, F. and Donegan, h. 1995 Comparison of prioritization techniques using interhierarchy map. Journal of operations Research Society 46(4): 492-498

[4] Feng Kong and Hongyan Liu, 2005, Applying fuzzy analytic hierarchy process to evaluate success factors of E-Commerce, Institute For scientific Computing and Information, vol 1, Number 3-4, Pages 406-412.

[5] Gao, Z. F., Wang, N., Xu, y., Shan, H., Li, X-D: Astron. Nachr. 336(2015) 866.

[6] Gao, Z. F., Li, X-D., Wang, N., Yuan, J.P., Wang, P., Wang. Peng, Q.H, Du, Y.J.: Mon. Not. R. Astron. Soc. 456(2016)55.

[7] Hwang, C.L. and Yoon, K. 1998. Multiple Attribute Decision Making Method and Applications, Springer, Berlin Heidelberg. 
[8] Jana Krejci, Ondrej Pavlacka and Jana Talasova, 2016 A Fuzzy extension of analytic Hierarchy Process based on the constrained fuzzy arithematic, Springer science+business Media New York.

[9] Levary, R.R and Wan, K. 1998. A simulation Approach for Handling Uncertainties in the analytical Hierarchy Process. European Journal of Operations Research Society 106(1), 116-122.

[10] Linkov,I. Welle, P. Loney, D. Tkachuk, A. Canis, A. J.B. Kim and T. Bridges, Use of Multicriteria Decision Analysis to support weight of Evidence Evaluation, Risk Anal. 31(8) (2011) 1211-1225

[11] Maji.P.K.Biswas.R and Roy A.R, 2003 soft set theory, computer and mathematics with Applications 555-562.

[12] Mignel, A.O.B et al., 2016. An AHP-Topsis integrated model for selecting the most appropriate tomography equipment, International Journal of Information \& Technology \& Decision Making, Vol. 15 (2016)

[13] Molodstov.D., 1999 soft set theory-First results Computers and Mathematics with applications, 19-31.
[14] Ramik, J. (2015). Isomorphism between fuzzy pair wise comparison matrices. Fuzzy Optimization and decision making, 14(2), 199-209

[15] Sabir Hussain and Mohammad Ahmad Alghamdi, 2012, On Soft Expert Topological Spaces, Springer Plus.

[16] Shabir, M and Naz, M., 2011 on soft Topological Spaces, Computers and Mathematics with Applications 61(7) 1786-1799.

[17] Shawkat Alkhanzleh and Abdul Razak Saleh, 2011, Soft expert Set, Research article, Advances in Decision Sciences.

[18] Wand Y. J and Lee. H.S, 2007, Generalising TOPSIS for fuzzy multiple criteria group decision making, Journal of Computers and Mathematics, 53(1), 1762-1772.

[19] Xibei Yang., Tsau Young Lin., Jingyu Yang., Yan Li. and Dongiun Yu., "Combination of interval-valued fuzzy set and soft set, "Computers and mathematics with applications, 58(3): 521-527 (2009)

[20] Zulqarnain, M., and Saeed, M. 2016, An Application of Interval Valued Fuzzy Soft Matrix (IVFSM) In Decision Making,

Sci.Int.(Lahore),28(3),2261-2264,2016 